\title{
PRODUCCIÓN INVERNAL DE PERRITO DE CORTE (Antirrhinum majus L.) EN EL VALLE DE MÉXICO
}

\section{WINTER PRODUCTION OF SNAPDRAGON (Antirrhinum majus L.) IN THE VALLEY OF MÉXICO}

\author{
Adriana Miranda Medina ${ }^{1}$, Jorge A. Gutiérrez Espinosa ${ }^{1 *}$, Ma. Teresa Colinas León ${ }^{2}$, \\ Lourdes Arévalo Galarza ${ }^{1}$ y E. Araceli Gaytán Acuña ${ }^{1}$
}

\begin{abstract}
${ }^{1}$ Programa de Recursos Genéticos y Productividad, Colegio de Postgraduados, Campus Montecillo. Carretera México-Texcoco, Km. 36.5. 56230, Monteci1lo, Texcoco, Estado de México. Tel (595)9520200 Ext 1113. ${ }^{2}$ Departamento de Fitotecnia, Universidad Autónoma Chapingo. Carretera Federal MéxicoTexcoco, Km. 38.5. 56260, Chapingo, Texcoco, Estado de México.
\end{abstract}

*Autor para correspondencia (jage@colpos.mx)

\section{RESUMEN}

El desarrollo y calidad comercial de cuatro cultivares de perrito (Antirrhinum majus L.) del Grupo I ('Flamingo', 'Yosemite Pink', 'White-Ivory' y 'EuroRose') y dos del Grupo IV ('Rose' y 'White') se evaluaron en un invernadero de cristal durante el ciclo de invierno 2004 en Montecillo, México. Los cultivares Grupo IV, produjeron tallos con $24 \%$ más de peso y $8 \%$ más de longitud que los del Grupo I, por lo que el Grupo IV logró mayor índice de calidad comercial $\left(0.68 \mathrm{~g} \mathrm{~cm}^{-1}\right)$ que el Grupo I $\left(0.53 \mathrm{~g} \mathrm{~cm}^{-1}\right)$. La calidad comercial "Especial", considerada la más alta por la Sociedad Americana de Floristas, se obtuvo en todos los tallos del Grupo IV, mientras que sólo 66.5 \% de los tallos producidos por el Grupo I lograron esta clasificación. Es factible la producción de invierno con calidad comercial "Especial" de los cultivares del Grupo IV en invernaderos sin calefacción del valle de México, aunque requieren 25 d más para alcanzar la madurez comercial que los cultivares del Grupo I.

Palabras clave: Antirrhinum majus, calidad comercial, invernadero, producción de invierno.

\section{SUMMARY}

Growth and commercial quality of four snapdragon cultivars (Antirrhinum majus L.) from Group I ('Flamingo', 'Yosemite Pink', 'White-Ivory' and 'EuroRose') and two from Group IV ('Rose' and 'White'), were evaluated under glass greenhouse conditions during the Winter of 2004, in Montecillo, México. Results showed that the flowering stems of Group IV were $24 \%$ heavier and $8 \%$ longer than those from Group I, so that the Group IV reached a higher quality index $\left(0.68 \mathrm{~g} \mathrm{~cm}^{-1}\right)$ than Group I $\left(0.53 \mathrm{~g} \mathrm{~cm}^{-1}\right)$. Moreover, when flower quality was assessed according to the Society of the American Florists standards, all the flowering stems produced by Group IV reached the "Special" quality standard which is the highest possible classification for a cut snapdragon stem; while about $66.5 \%$ of the flowering stems produced by Group I reached this quality. Winter production of high quality flowering shoots of Group IV cultivars in the Valley of México is possible in non-heated greenhouses, although they need $25 \mathrm{~d}$ to reach the commercial harvest stage than Group I.

Index words: Antirrhinum majus, commercial quality, greenhouse, winter production.

\section{INTRODUCCIÓN}

El "antirrino o perrito" (Antirrhinum majus L.) es cultivado en México en la industria del paisajismo ornamental (INEGI, 1998), mientras que su uso en la producción de flor de corte es incipiente y representa una opción con potencial (Gutiérrez, 2005; Miranda y Gutiérrez, 2005). El cultivo anual de esta especie es posible debido al mejoramiento genético de cultivares clasificados en cuatro grupos comerciales de respuesta a luz y temperatura (Dole y Wilkins, 2005). Esta clasificación permite a los productores elegir cultivares y producir tallos con mejor calidad comercial, de acuerdo con las condiciones climáticas prevalecientes (Cockshull, 1985; Larson, 1992). Los cultivares producidos en el ciclo otoño-invierno (Grupos I y II) requieren temperaturas de $14-25 / 8-15{ }^{\circ} \mathrm{C}$ día/noche y fotoperiodos cortos $(<10 \mathrm{~h})$, y desarrollan pocas hojas axilares por debajo de la espiga floral. En cambio, los cultivares producidos en el ciclo primavera-verano (Grupos III y IV) requieren temperaturas de $18-28 / 14-18{ }^{\circ} \mathrm{C}$ día/noche y fotoperiodos largos $(>10 \mathrm{~h})$, condiciones que les permiten desarrollar mayor número de hojas axilares antes de la madurez comercial (Cockshull, 1985).

Los tallos de los cultivares del Grupo IV (Potomac cvs. 'White' y 'White/Ivory') tienen más área foliar, longitud de tallo floral y producción de biomasa que los cultivares del Grupo I (Maryland cvs. 'White' y 'White/Ivory'), diferencia que se debe a su mayor capacidad fotosintética (Gutiérrez-Espinosa, 2003; Com. personal) ${ }^{1}$. Estas diferencias en capacidad fotosintética pueden

\footnotetext{
${ }^{1}$ Gutiérrez-Espinosa J A (2003) Growth, development and photosynthesis of the snapdragon (Antirrhinum majus L.) canopy during different seasons. Ph.D. Thesis. Department of Plant Agriculture. Horticultural Science. University of Guelph. Ont., Canada. 330 p.
} 
conducir a ventajas comerciales en la producción de invierno de perrito porque los cultivares del Grupo I son más eficientes que los del Grupo IV en el intercambio de carbono en respuesta a menores puntos de compensación de luz (18 y $\left.32 \mu \mathrm{mol} \mathrm{m}^{-2} \mathrm{~s}^{-1}\right)$ (Gutiérrez et al., 2005). En consecuencia, los cultivares de los Grupos I y II son más eficientes fotosintéticamente $\left(2.40\right.$ y $1.95 \mathrm{~mol} \mathrm{CO}_{2} \mathrm{~mol}$ $\mathrm{RFA}^{-1} \times 10^{-2}$, respectivamente), lo cual justifica producirlos en invierno en zonas por arriba de los $38^{\circ} \mathrm{LN}$, con bajas temperaturas y fotoperiodos cortos (Laughner y Corr, 1997). La producción de invierno en zonas por abajo de los $38{ }^{\circ} \mathrm{LN}$ requiere cultivares de los Grupos III y IV porque es cuando se presentan fotoperiodos mayores a 10 h (Laughner y Corr, 1997).

Estudios realizados con cultivares de los Grupos III y IV ('Colorado', 'Dark Star', 'North Carolina', 'Oklahoma', 'Panama', 'Tampico', 'Utah', 'White Skies') y dos de la serie Potomac ('White' y 'Yellow') crecidos en condiciones óptimas de riego, mostraron una alta correlación $(\mathrm{r}=0.82)$ entre la densidad estomática y la transpiración diaria, con valores promedio de 184.6 estomas $\mathrm{mm}^{-2}$ y niveles de transpiración de $43.59 \mathrm{~g} / \mathrm{planta}$ (Rutland et al., 1987). La relación lineal entre la radiación total y la transpiración diaria ( $r=0.99$; Rutland, 1972), también es un aspecto importante relacionado con la producción y calidad de los diferentes grupos de perrito.

En México no se ha investigado el desarrollo, la calidad comercial, ni la vida de florero de cultivares de perrito para corte. Por ello en el presente estudio se comparó la producción de tallos, calidad comercial y vida en poscosecha de los Grupos I y IV de perrito de corte, bajo condiciones de invernadero durante el ciclo otoñoinvierno.

\section{MATERIALES Y MÉTODOS}

El experimento se llevó a cabo durante el invierno 2004 en un invernadero de vidrio, en Montecillo, Texcoco, Edo. de México (2250 m de altitud; 19¹9' LN y $98^{\circ}$ $53^{\prime}$ LO). Se utilizaron semillas de seis cultivares (PanAmerican Seeds Co., IL, USA), cuatro provenientes del Grupo I ('Flamingo', 'Yosemite Pink', 'White/Ivory' serie Maryland y 'EuroRose' serie Winter), y dos del Grupo IV ('Rose' y 'White' serie Potomac), que se sembraron el 9 de noviembre del 2004, en charolas de 128 cavidades; el trasplante se hizo el 12 de diciembre del mismo año en macetas de $15.2 \mathrm{~cm}$ (Polietilenos del Sur ${ }^{\circledR}$, Morelos, México) cuando las plántulas tenían dos pares de hojas, y se colocó una plántula por contenedor. Como sustrato se utilizó la mezcla comercial Promix PGX, No. 3 (Les tourbières Premier LTÈE, Quebec, Canadá). Cada semana se aplicaron $200 \mathrm{mg} \mathrm{L}^{-1}$ de la fórmula comercial
N-P-K (Peters, General Purpose, Scotts Co., OH, USA) a través del agua de riego. Adicionalmente, $60 \mathrm{~d}$ después de la siembra se aplicó nitrato de calcio $\left(\mathrm{Ca}\left(\mathrm{NO}_{3}\right)_{2}\right)$ a razón de $200 \mathrm{mg} \mathrm{L}^{-1}$ de Ca (1 g L de solución). Los riegos se aplicaron manualmente cada $2 \mathrm{~d}$ con el agua corriente colocada a pie de llave dentro del invernadero, con un bastón de riego y una cebolla de aspersión (SunMate, Orbit, UT, USA). Se utilizó malla de tutoreo plástica (12.5 x $12.5 \mathrm{~cm}$; Polietilenos del Sur, Jiutepec, Morelos, México) a partir de los $30 \mathrm{~d}$ después del trasplante. Los tallos se cortaron cuando se alcanzó la madurez comercial, cuando dos terceras partes de las flores de la espiga floral se encontraban abiertas (Gutiérrez-Espinosa; 2003; opns. cit.).

La temperatura $\left({ }^{\circ} \mathrm{C}\right)$, humedad relativa $(\%)$ y luminosidad (lumens $\mathrm{cm}^{-2}$ ) dentro del invernadero se registraron con un data logger HOBO modelo H8 (Onset Co. MA., USA), el cual se colocó a $50 \mathrm{~cm}$ de altura del suelo y se programó para registrar lecturas cada 10 min. También se obtuvo la radiación total $\left(\mathrm{W} \mathrm{m}^{-2}\right.$; Li-Cor ${ }^{\circledR} \mathrm{LI}-200 \mathrm{SZ}{ }^{\circledR}$, NE, USA) y la temperatura del aire $\left({ }^{\circ} \mathrm{C}\right.$; Argus TN21/S) al exterior del invernadero, de la estación climatológica (GroWeather, Davis Instruments Co., CA, USA) del Colegio de Postgraduados.

Para evaluar el desarrollo del cultivo se midió: biomasa total fresca y seca $(\mathrm{g})$, longitud total de tallo $(\mathrm{cm}) \mathrm{y}$ longitud de espiga floral $(\mathrm{cm})$, número de hojas axilares, días a cosecha, e índice de calidad (IC, $\mathrm{g} \mathrm{cm}^{-1}$ de tallo). Se consideró la calidad comercial de los tallos de acuerdo con los estándares establecidos por la Sociedad Americana de Floristas (SAF; Cuadro 1). Además, se determinó la densidad estomática con la técnica de impresión en pegamento liquido, en hojas axilares completamente desarrolladas y expandidas de la parte media del tallo (Martin y Stitmart, 2005). El conteo de estomas se hizo en las porciones basal y media del área abaxial y adaxial de las hojas, con un Foto-Microscopio III Carl Zeiss modelo 68020 equipado con una cámara Pixera professional.

Cuadro 1. Estándar de la clasificación comercial de tallos florales de perrito (Antirrhinum majus) de la Sociedad Americana de Floristas (SAF).

\begin{tabular}{lccc}
\hline & \multicolumn{3}{c}{ Criterios de Clasificación } \\
\cline { 2 - 4 } Clasificación & $\begin{array}{c}\text { Biomasa total } \\
\text { fresca del } \\
\text { tallo floral }(\mathrm{g})\end{array}$ & $\begin{array}{c}\text { Longitud del } \\
\text { tallo floral } \\
(\mathrm{cm})\end{array}$ & $\begin{array}{c}\text { Número de } \\
\text { floretes abiertos } \\
\text { en espiga (num) }\end{array}$ \\
\hline Especial & $71-113$ & $>91$ & $>15$ \\
Sofisticada & $43-70$ & $61-76$ & $9-12$ \\
Extra & $29-42$ & $46-61$ & $6-9$ \\
Primera & $14-28$ & $<46$ & $<6$ \\
\hline
\end{tabular}

Fuente: Larson RA (1992). Introduction to Floriculture.

El diseño experimental fue uno de bloques al azar, con 90 plantas por bloque experimental y cuatro bloques. Se establecieron plantas adicionales en la periferia de los 
bloques experimentales para contrarrestar el efecto de orilla. La diferencia entre medias de tratamientos se analizó mediante la prueba Tukey $(\alpha=0.05)$. Los grupos de respuesta se consideraron como efectos confundidos, por lo que se hicieron análisis de contrastes ortogonales para determinar diferencias entre cultivares por su respuesta grupal. Los análisis estadísticos se hicieron con el paquete SAS versión 8.0 (SAS Institute, 1999).

La ganancia de biomasa fresca durante la vida de florero se determinó después de cortar los tallos a $60 \mathrm{~cm}$ por debajo de la espiga floral, sin eliminar las hojas existentes en los $10 \mathrm{~cm}$ del extremo distal del tallo (Besmer y Koide, 1999). Posteriormente, los tallos fueron colocados en tubos de polivinil (30 y $5 \mathrm{~cm}$ de diámetro) con $250 \mathrm{~mL}$ de agua destilada. La ganancia en peso fue evaluada diariamente con el peso individual de los tallos y del agua contenida en los tubos, con una balanza digital A\&D modelo EY-2200A. La ganancia en peso y el error estándar por tratamiento se graficaron con el paquete GraphPad Prism v.4.

\section{RESULTADOS Y DISCUSIÓN}

\section{Ambiente de desarrollo}

Al exterior del invernadero la luminosidad y temperatura en la etapa final del desarrollo del cultivo fueron superiores a $900 \mu \mathrm{mol}$ fotón $\mathrm{m}^{-2} \mathrm{~s}^{-1}$ (RFA) y $13{ }^{\circ} \mathrm{C}$ en promedio, mientras que al interior del invernadero la luminosidad se mantuvo por encima de $600 \mu$ mol fotón $\mathrm{m}^{-2} \mathrm{~s}^{-1}$ (RFA), con temperaturas mínimas de $5^{\circ} \mathrm{C}$ y promedio de $16{ }^{\circ} \mathrm{C}$, durante el ciclo de desarrollo. Karlsson y Werner
(2001) consideran a la temperatura promedio diaria como determinante en la tasa de desarrollo del cultivo, mientras que el fotoperiodo afecta los procesos internos de desarrollo (Catley et al., 2002; Jackson y Thomas, 1997) y determinan la iniciación a floración (Khattak y Pearson, 2005). El uso de invernaderos fríos en el Valle de México, con reducido o nulo calentamiento (Noto y Romano, 1989), es cada vez mas frecuente debido al aumento del precio de los combustibles. Un creciente número de productores en la temporada de invierno recurren al desarrollo de cultivos con menores requerimientos de temperatura. Bajo estas condiciones, el cultivo de perrito de corte es una alternativa con potencial (Gutiérrez-Espinosa, 2005).

\section{Variables de desarrollo}

Los cultivares 'Yosemite Pink' (75.4 g) y 'EuroRose' $(75.9 \mathrm{~g})$ produjeron los tallos más pesados dentro del Grupo I; no obstante, su biomasa fresca total fue hasta 15 g menor que la de los cultivares del Grupo IV (88.5 y $91.3 \mathrm{~g}$ ). 'Yosemite Pink' fue el único cultivar del Grupo I que alcanzó una longitud de tallo total similar a los tallos de los cultivares del Grupo IV, pero en el IC promedio se observó un incremento de $22 \%$ en los cultivares del Grupo IV (0.68 $\left.\mathrm{g} \mathrm{cm}^{-1}\right)$ comparado con los del Grupo I (0.53 $\left.\mathrm{g} \mathrm{cm}^{-1}\right)$. Por otro lado, los tallos del cultivar 'Yosemite Pink' desarrollaron la espiga floral de mayor longitud $(28.9 \mathrm{~cm})$, inclusive mayor que la espiga de los cultivares del Grupo IV (24.8 y $25.9 \mathrm{~cm})$. Los cultivares del Grupo I produjeron en promedio 10 hojas axilares menos que los cultivares del Grupo IV (Cuadros 2 y 3 ).

Cuadro 2. Variables de desarrollo del cultivo de perrito (Antirrhinum majus) de corte, al crecer en invernadero, en los grupos de floración (Grupos I y IV) durante el invierno 2004. Montecillo, México.

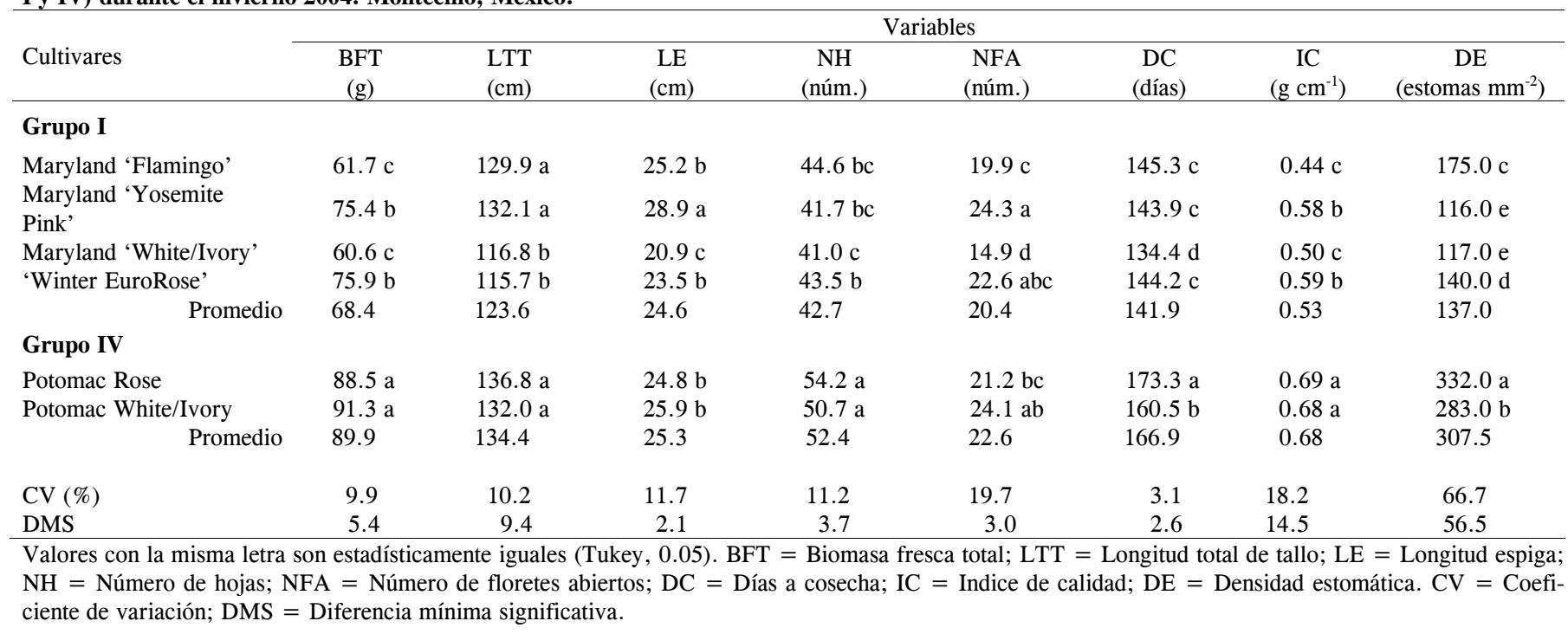


La densidad estomática varió en los cultivares del Grupo I (Cuadro 2), aunque en promedio fue menos de la mitad (137.0 estomas $\mathrm{mm}^{-2}$ ) que en los cultivares del Grupo IV (137.0 y 307.5 estomas $\mathrm{mm}^{-2}$ ) (Cuadro 3). Los cultivares del Grupo IV ('Colorado', 'Dark Star', 'North Carolina', 'Oklahoma', 'Panama', 'Tampico', 'Utah', 'White Skies' y dos de la serie Potomac cvs. 'White', 'Yellow') muestran densidades estomáticas similares (Rutland, 1972; Rutland et al., 1987). Se ha señalado que la mayor densidad estomática de los cultivares del Grupo IV sirve para adaptarse a los niveles de luminosidad y temperatura mayores durante el verano (McDaniel y Miller, 1976; Rutland et al., 1987). Es importante hacer notar que ningún estudio realizado en México se refiere a diferencias en densidad estomática entre cultivares de ambos grupos, a pesar de que esta diferencia es un indicador de que el perrito se ha adaptado a ambientes extremos. Los cultivares del Grupo IV produjeron mayor biomasa fresca por longitud de tallo producido $\left(\mathrm{g} \mathrm{cm}^{-1}\right)$, y presentaron tallos más pesados y con mayor número de hojas axilares que los cultivares del Grupo I (Cuadros 2 y 3 ).

Cuadro 3. Contrastes ortogonales de las variables de desarrollo en los Grupos I y IV del cultivo de perrito (Antirrhinum majus) de corte durante el invierno 2004 en Montecillo, México.

\begin{tabular}{lcc}
\hline Variables de desarrollo & Grupo I vs. Grupo IV & $\operatorname{Pr}>\mathrm{F}$ \\
\hline BFT (g) & 68.4 vs $89.9 * *$ & 0.0001 \\
LTT (cm) & 123.6 ss $134.4 * *$ & 0.0077 \\
LE (cm) & 24.6 vs $25.3 \mathrm{~ns}$ & 0.6088 \\
NH (num.) & 42.7 vs $52.4 * *$ & 0.0104 \\
NFA (num) & 20.42 vs $22.65 \mathrm{~ns}$ & 0.8836 \\
DC (días) & 141.9 vs $166.9 * *$ & 0.0001 \\
IC $\left(\mathrm{g} \mathrm{cm}^{-1}\right)$ & 0.53 vs $0.68 * *$ & 0.0069 \\
DE (estomas $\left.\cdot \mathrm{mm}^{-2}\right)$ & 137.0 vs $307.5 * *$ & 0.0001 \\
\hline
\end{tabular}

$\mathrm{ns}$, ** No significativo y significativo a nivel de $\mathrm{P} \leq 0.01$ respectivamente; $\mathrm{BFT}=$ Biomasa fresca total; LTT $=$ Longitud total de tallo; $\mathrm{LE}=$ Longitud espiga; $\mathrm{NH}=$ Número de hojas; $\mathrm{NFA}=$ Número de floretes abiertos; $\mathrm{DC}=$ Días a cosecha; $\mathrm{IC}=$ Índice de calidad y DE = Densidad estomática.

La longitud de la espiga floral y el número de flores abiertas en la espiga fueron las únicas variables que mostraron características similares entre cultivares de ambos grupos. Los del Grupo I alcanzaron la madurez comercial $25 \mathrm{~d}$ antes que los cultivares del Grupo IV, con promedios de 141.6 y 166.9 d, respectivamente (Cuadro 3). Este resultado es congruente con las condiciones de invernadero frío en donde las temperaturas mínimas al interior fueron inferiores a $10{ }^{\circ} \mathrm{C}$, suficientes para retrasar el desarrollo de los cultivares del Grupo IV. La altura de tallos y la distancia de entrenudos son afectados cuando cambia la calidad de la luz (Khattak y Pearson, 2005; Munir et al., 2004a). Asimismo, la longitud de la espiga floral, el número y la velocidad de apertura de las flores son controlados por la temperatura dominante en el cv. 'Chimes White' (Munir et al., 2004b). Según Gutiérrez-Espinosa
(2005), los cultivares de perrito Maryland 'White', Maryland 'White/Ivory', Potomac 'White' y Potomac 'White/Ivory' cultivados en invierno ( 15 moles $\left.\mathrm{m}^{-2} \mathrm{~d}^{-1}\right)$ tienen tallos más cortos, menor número de hojas principales y menor área foliar que los cultivados en verano (42.5 moles $\left.\mathrm{m}^{-2} \mathrm{~d}^{-1}\right)$.

\section{Calidad comercial}

Sullivan y Pasian (2000) demostraron que conforme más biomasa fresca se acumula, mayor longitud tendrá el tallo producido; no obstante, en el presente estudio sólo los cultivares del Grupo IV mostraron esta relación. En los cultivares del Grupo I su menor producción de biomasa fresca se reflejó en un bajo índice de calidad (Cuadro 2), pues aunque los tallos alcanzaron la altura deseable sus índices de calidad (IC) fueron inferiores a los del Grupo IV; las comparaciones mediante contrastes ortogonales (Cuadro 3) permitieron confirmar que los cultivares del Grupo IV; superaron a los del Grupo I en el IC. Los resultados de la calidad comercial SAF mostró que todos los tallos del Grupo IV alcanzaron la calidad "Especial", que es la más alta para el manejo comercial de tallos de perrito; en contraste, sólo $66.5 \%$ de los tallos provenientes del Grupo I obtuvieron la calidad "Especial" y el resto se ubicó en la calidad "Sofisticada" (Cuadros 1 y 4).

Cuadro 4. Calidad comercial (según la Sociedad Americana de Floristas, SAF) de la producción invernal de seis cultivares de perrito (Antirrhinum majus) de corte, en dos grupos de precocidad a floración (Grupos I y IV). Montecillo, México.

\begin{tabular}{|c|c|c|c|}
\hline Cultivares & \multicolumn{2}{|c|}{$\begin{array}{l}\text { Calidad SAF } \\
\text { (\% Tallos })\end{array}$} & $\begin{array}{c}\mathrm{IC} \\
\left(\mathrm{g} \mathrm{cm}^{-1} \text { tallo }\right)\end{array}$ \\
\hline & "Especial" & "Sofisticada" & \\
\hline \multicolumn{4}{|l|}{ Grupo I } \\
\hline Maryland 'Flamingo' & $91 \mathrm{a}$ & $9 \mathrm{~b}$ & 0.48 \\
\hline $\begin{array}{l}\text { Maryland 'Yosemite } \\
\text { Pink' }\end{array}$ & $72 \mathrm{a}$ & $28 \mathrm{~b}$ & 0.57 \\
\hline Maryland 'White/Ivory' & $19 \mathrm{~b}$ & $81 \mathrm{a}$ & 0.52 \\
\hline Winter 'EuroRose' & $84 \mathrm{a}$ & $16 \mathrm{~b}$ & 0.67 \\
\hline Promedio & 66.5 & 33.5 & 0.56 \\
\hline \multicolumn{4}{|l|}{ Grupo IV } \\
\hline Potomac 'Rose' & 100 & - & 0.69 \\
\hline Potomac 'White/Ivory' & 100 & - & 0.68 \\
\hline Promedio & 100 & - & 0.68 \\
\hline
\end{tabular}

Valores con la misma letra entre columnas son estadísticamente iguales (Tukey, 0.05); IC = Índice de calidad.

\section{Ganancia en biomasa fresca en postcosecha}

La absorción de agua por los tallos del Grupo I fue continua hasta el octavo día después de cortados. Los cultivares de la serie Maryland incrementaron hasta en $30 \%$ su biomasa fresca, mientras que el cv. 'EuroRose' de la serie Winter tuvo la menor absorción de agua, la cual además disminuyó después del octavo día (Figura 1A). Una elevada capacidad de absorción de agua es indicativa de un balance hídrico positivo, porque la absorción de 
agua es mayor o igual que la transpiración, condición necesaria para una larga vida de florero (van Meeteren et al., 2005).
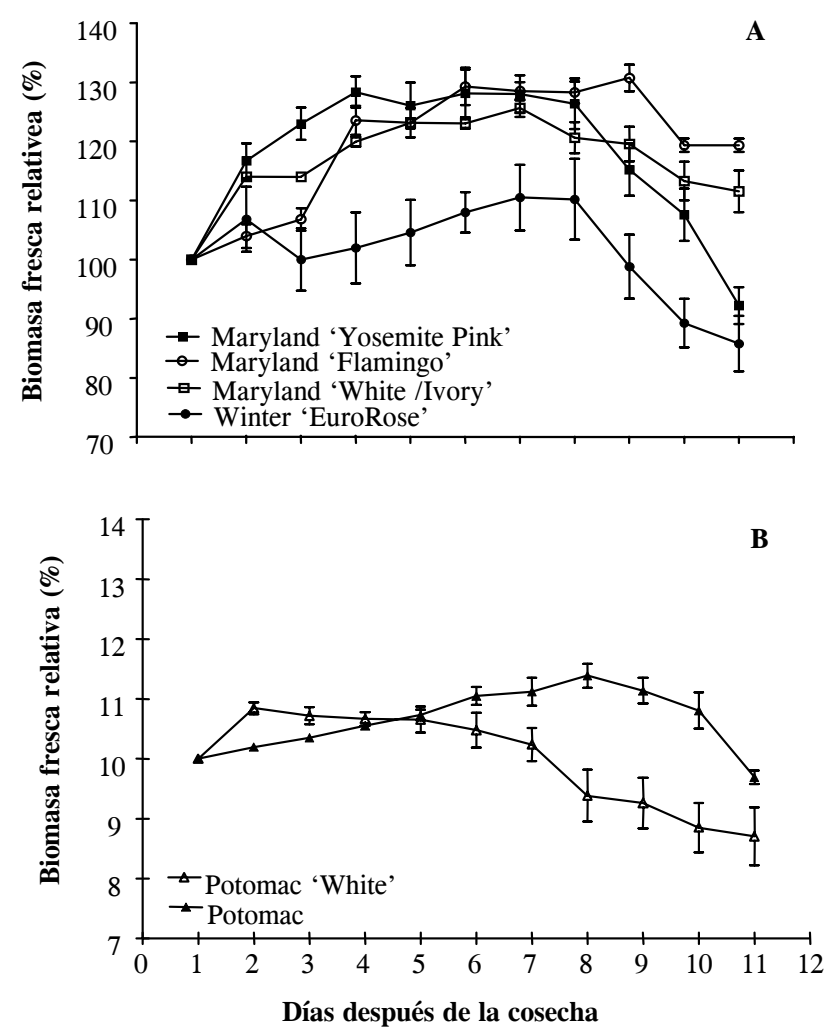

Figura 1. Ganancia de biomasa fresca relativa (\%) en cultivares del Grupo I (A) y del Grupo IV (B) de perrito (Antirrhinum majus) de corte colocados en agua durante la evaluación postcosecha. Las barras representan el error estándar de la media $(n=10)$.

Los cultivares del Grupo IV absorbieron menos agua (10-15\%) que los del Grupo I (Figura 1B). A partir del segundo día después del corte, la absorción de agua de 'White/Ivory' de la serie Potomac descendió continuamente lo que generó marchitamiento y afectó la vida de florero, con pérdidas de biomasa fresca superiores a $10 \%$ al octavo día. Según Zencirkiran (2002), cuando las flores cortadas tienen pérdidas superiores a $8 \%$ de su peso inicial, difícilmente recuperan la turgencia y presentan marchitez. Al respecto, los cultivares 'EuroRose' y 'Yosemite' perdieron la turgencia a los $11 \mathrm{~d}$, 'White/Ivory' serie Potomac se marchitó entre los 8 y 9 d, mientras que los cultivares de la serie Maryland ('Flamingo' y 'White/Ivory') se mantuvieron turgentes hasta los $12 \mathrm{~d}$ (Figura 1 A y B). En este trabajo los cultivares del Grupo I tuvieron mayor capacidad de absorción de agua pero también menores índices de calidad y menor desarrollo de la planta. En otro trabajo se encontró que los cultivares de Zinnia elegans presentan una relación inversa entre la capacidad de absorción de agua y la dimensión de los vasos del xilema (número, longitud y diámetro), lo que significará que los tallos menos vigorosos poseen mayor capacidad de absorción de agua (Twumasi et al., 2005). Por su parte, van Meeteren et al. (2005) consideran que la habilidad de los tallos florales para restablecer un balance hídrico positivo depende de las características de los vasos del xilema las cuales están determinadas por la especie, cultivar y condiciones de crecimiento. En este estudio, en cambio, los cultivares del Grupo IV, cuyos tallos presentaron mayor vigor, fueron los que tuvieron menor capacidad de absorción de agua, en comparación con los del Grupo I (Cuadros 2 y 3). Debido a esta controversia, es recomendable hacer más estudios de postcosecha en esta especie.

\section{CONCLUSIONES}

Los cultivares 'Rose' y 'White' de la serie Potomac del Grupo IV, tuvieron mayor calidad comercial, lo que, hace factible su cultivo en invernaderos sin calefacción. Estos materiales prolongaron hasta $25 \mathrm{~d}$ su estadía en el invernadero, tiempo que podría reducirse mediante calentamiento del invernadero. La mayor densidad estomática en los cultivares del Grupo IV le confirió mayor adaptabilidad al frío invernal, en comparación con los del Grupo I. A pesar de que los cultivares del Grupo I ganaron más biomasa fresca en vida poscosecha, ello no se asoció con menor marchitamiento de tallos.

\section{AGRADECIMIENTOS}

Al CONACYT (Consejo Nacional de Ciencia y Tecnología) y al COMECYT (Consejo Mexiquense de Ciencia y Tecnología) por el apoyo económico, y al Departamento de Fitotecnia de la Universidad Autónoma Chapingo por las facilidades otorgadas para la realización de este estudio.

\section{BIBLIOGRAFÍA}

Besmer Y L, T R Koide (1999) Effect of mycorrhizal colonization and phosphorus on ethylene production by snapdragon (Antirrhinum majus L.) flowers. Plant and Soil 9:161-166.

Cockshull K E (1985) Antirrhinum majus. In: The Handbook of Flowering, Vol. 1. A H Halevy (ed). CRC Press, Boca Raton, FL. pp:476-481.

Dole J M, H F Wilkins (2005) Antirrhinum. In: Floriculture Principles and Species. D Yarnell, K Yehle, L Dalberg (eds). Pearson Prentice Hall. New Jersey, USA. pp:284-294.

Gutiérrez-Espinosa J A (2005) Cultivo de Perrito: Una alternativa con potencial, Parte 1. TecnoAgro 6:42-44.

Gutiérrez-Espinosa J A, R R Cloutier, N Ortiz-Uribe, D L Evangelos, B Grodzinski (2005) Canopy and leaf photosynthesis in cultivars of Antirrhinum majus L. acclimated for crop production in low or high light conditions. In: Photosynthesis Fundamental Aspects to Global Perspectives. A Van der Est, D 
Bruce (eds). Allen Press Inc. Lawrence, KS. USA. pp:10611063.

Instituto Nacional de Estadística Geografía e Informática, INEGI (1998) La Horticultura Ornamental en México. INEGI, México. pp:42, 61, 78.

Jackson S, B Thomas (1997) Photoreceptors and signals in the photoperiodic control of development. Plant Cell Environ. 20:790795.

Karlsson M G, J W Werner (2001) Temperature after flower initiation affects morphology and flowering of cyclamen. Sci. Hortic. 91:357-363.

Khattak A.M, S Pearson (2005) Light quality and temperature on antirrhinum growth and development. J. Zhejiang Univ. Sci. 6B:119-124.

Larson R A (1992) Introduction to Floriculture. Academic Press Inc. Raleigh, NC, USA. 551 p.

Laughner L, B Corr (1997) Snapdragons: Formula for success. In Culture Notes. Grower Talks Mag. (Sept.):57-58.

Martin W J, D P Stitmart (2005) Stomatal density in Antirrhinum majus L., inheritance and trends with development. HortScience 40:1252-1258.

McDaniel G L, M G Miller (1976) Transpiration of snapdragon under southern greenhouse conditions. HortScience 11:366-368.

Miranda-Medina A, J A Gutiérrez-Espinosa (2005) Cultivo de Perrito: Una alternativa con potencial, Parte 2. TecnoAgro 6:2628

Munir M, M Jamil, J Baloch, K R Khattak (2004a) Impact of Light intensity on flowering time and plant quality of Antirrhinum majus L. cultivar 'Chimes white'. J. Zhejiang Univ. Sci. 5:400-405

Munir M, M Jamil, J Baloch, K R Khattak (2004b) Growth and flowering of Antirrhinum majus L. under varying temperatures. Int. J. Agric. Biol. 6:173-178.

Noto G, D Romano (1989) Timing of Snapdragon Antirrhinum majus L. in cold greenhouse cultivation. Acta Hort. 246:175-181.

Rutland R B (1972) Transpiration of Antirrhinum majus L. in relation to radiant energy in the greenhouse. HortScience 7:39-40.

Rutland R B, H L Chang, J E Pallas Jr (1987) Stomatal density of Snapdragon as a possible determinant of transpiration. HortScience 22:599-601.

SAS Institute (1999) SAS User's Guide: Statistics. Version 8.0. SAS Institute, Inc. Cary, NC, USA. 956 p.

Sullivan K J, C C Pasian (2000) Evaluation of two growing systems for cut snapdragon production: tray vs ground bed. HortScience 35:25-27

Twumasi P, W van Ieperen, E J Woltering, A M C Emons, J H N Schel, J F H Snel, U van Meeteren, D van Marwijk (2005) Effects of water stress during growth on xylem anatomy, xylem functioning and vase life in three Zinnia elegans cultivars. Acta Hort. 669:303-311.

van Meeteren U, A van Gelder, W van Ieperen (2005) Effect of growth conditions on postharvest rehydration ability of cut chrysanthemum flowers. Acta Hort. 669:287-295.

Zencirkiran M (2002) Cold storage of Fressia refracta 'Cordula'. New Zealand J. Crop Hort. Sci. 30:171-174. 